\title{
Polariton relaxation and polariton nonlinearities in nonresonantly cw-pumped III-nitride slab waveguides
}

\author{
J. Ciers $\odot,{ }^{1, *}$ D. D. Solnyshkov $\odot,{ }^{2,3}$ G. Callsen $\odot,{ }^{1, \dagger}$ Y. Kuang $\odot,{ }^{1}$ J.-F. Carlin, ${ }^{1}$ G. Malpuech, ${ }^{2}$ R. Butté $\odot,{ }^{1}$ and N. Grandjean ${ }^{1}$ \\ ${ }^{1}$ Institute of Physics, École Polytechnique Fédérale de Lausanne (EPFL), CH-1015 Lausanne, Switzerland \\ ${ }^{2}$ Institut Pascal, PHOTON-N2, University Clermont Auvergne, CNRS, SIGMA Clermont, F-63000 Clermont Ferrand, France \\ ${ }^{3}$ Institut Universitaire de France (IUF), F-75231 Paris, France
}

(Received 17 June 2020; revised 30 September 2020; accepted 1 October 2020; published 19 October 2020)

\begin{abstract}
Polariton lasers are mostly based on planar cavities. Here we focus on an alternative configuration with slab waveguide modes strongly coupled to excitons confined in GaN/AlGaN quantum wells. We study experimentally and theoretically polariton relaxation at temperatures ranging from 4 to $200 \mathrm{~K}$. We observe a good robustness of the lower polariton population peak energy position against temperature changes due to a balance between the shift of the exciton energy and the change in the normal mode splitting, a promising feature for future applications such as lasers and amplifiers where a small temperature drift in the emission wavelength is a desired asset. Finally, at $T=4 \mathrm{~K}$ we observe the signature of polariton nonlinearities occurring in the continuous wave regime that are assigned to an optical parametric oscillation process.
\end{abstract}

DOI: 10.1103/PhysRevB.102.155304

\section{INTRODUCTION}

When the decay rates of both semiconductor excitons and confined optical modes are slower than their mutual rate of energy exchange (the Rabi splitting), the eigenmodes of the system are hybrid light-matter quasiparticles called exciton-polaritons or simply polaritons [1,2]. A wide range of polaritonic phenomena, often arising from their bosonic nature, can be triggered in planar microcavities [3] and in slab waveguides (WGs) [4]. These phenomena include various particular features of a quantum fluid of light, such as coherent emission without population inversion [5], parametric amplification [6,7], parametric oscillation [8], Bose-Einstein condensation [9], superfluidity [10], or soliton formation [11].

Beyond pure fundamental aspects, the remarkable physical phenomena ensuing from the mixed light-matter nature of polaritons open promising perspectives for the realization of low power consumption and small footprint active optical devices [12-17]. This is especially the case for slab WGs, which exhibit eigenmodes characterized by fast inplane propagation with group velocities on the order of $10^{7}$ $\mathrm{m} / \mathrm{s}$, whose geometry makes them more suitable for integration into photonic integrated circuits than their planar microcavity counterparts $[4,11,18-25]$.

\footnotetext{
*Present address: Department of Microtechnology and Nanoscience, Chalmers University of Technology, 41296 Gothenburg, Sweden.

$\dagger$ Present address: Institute of Solid State Physics, University of Bremen, 28359 Bremen, Germany.

Published by the American Physical Society under the terms of the Creative Commons Attribution 4.0 International license. Further distribution of this work must maintain attribution to the author(s) and the published article's title, journal citation, and DOI.
}

With the aim of realizing ever more complex polaritonic WG circuits, such as cascading devices, there is a need for a precise understanding of some key features such as the impact of temperature and optical pump power on polariton relaxation for this specific geometry but also whether observing polariton nonlinearities in the continuous wave $(\mathrm{cw})$ regime in other materials than the prototypical GaAs-based system is within reach. In this regard, developing platforms where polaritons exhibit a recognized robustness to their environment thanks to their large exciton binding energy $\left(E_{X}^{b}\right)$, like operation up to ambient temperature, is a highly desirable asset. In particular, such a statement does apply to the III-nitride (III-N) materials system, which was the first to sustain polariton nonlinearities at room temperature, with the observation of polariton lasing under pulsed excitation reported in both bulk and quantum well $(\mathrm{QW})$ based planar microcavities more than a decade ago [26,27]. So far, polariton relaxation dynamics, which has been studied in detail and is well understood in planar cavities [28-33], remains relatively unexplored in the slab WG geometry, especially as a function of lattice temperature. After the theoretical work [21] that pointed out the advantages of this configuration, an experimental confirmation of the possibility of amplification and polariton lasing in WGs [24], as well as a comparison of WG and planar cavity polariton lasers [34], were investigated under pulsed excitation using the $\mathrm{ZnO}$ platform. These works are complementary to those led on state-of-the-art GaAs WGs where numerous nonlinear optical features have been reported including darksoliton formation under $\mathrm{cw}$ excitation that exhibits enhanced polariton-polariton strength compared to experiments done with picosecond pulses [35] or modulational instabilities occurring in the pulsed regime like spatiotemporal continuum generation $[36,37]$. Let us note that all of those experiments take place in submillimeter long structures, which could be even further downscaled by applying longitudinal electric 
fields that increase even more the polariton-polariton interaction strength and thus nonlinear effects [25]. Those various aspects clearly evidence the potential of the WG geometry for the realization of on-chip compact nonlinear photonic systems relying on polaritons up to ambient temperature.

In all polariton systems, relaxation phenomena govern the polariton population distribution along polariton branches and the eventual emergence of nonlinearities triggered by the macroscopic occupancy of a given eigenstate by these bosonic particles. More than two decades ago, Tassone and co-workers showed that energy dissipation in planar microcavities, and hence polariton relaxation toward lower in-plane wave vector $\left(k_{/ /}\right)$states within the light cone, is essentially mediated by exciton-exciton and exciton-phonon interactions [28,29]. However, the decreasing excitonic character of lower polaritons (LPs) when moving toward lower energy states is known to quench the relaxation process, leading to a so-called polariton relaxation bottleneck $[28,38]$. The particle accumulation at the bottleneck, which is a drawback for planar cavities as it may prevent condensate formation in the ground state, becomes an important and useful asset in the WG geometry. Indeed, since there is no ground state anyway, an initial macroscopic population can only build up in the bottleneck region between the purely photonic and the purely excitonic states [21]. In such a situation, the position of the bottleneck is determined, as we should expect, by the balance between relaxation and decay processes.

In this work we focus on the relaxation dynamics of propagating LPs in III-N slab WGs under nonresonant cw optical pumping as a function of propagation distance for different temperatures and excitation power densities. This is done in a platform that combines reduced photonic disorder and a large $E_{X}^{b}$ value thanks to the use of high quality GaN/AlGaN multiple QWs (MQWs) grown on low dislocation density $c$-plane freestanding (FS) GaN substrate. Experimental data are supported by simulations based on the numerical solution of semiclassical Boltzmann equations for polaritons. Within this framework we also report polariton nonlinearities, which are well accounted for using the formalism of the GrossPitaevskii equation (GPE).

\section{SAMPLE AND MEASUREMENTS}

The structure under investigation is a slab WG that consists of a 130-nm-thick active region with 22 embedded $\mathrm{GaN} / \mathrm{Al}_{0.15} \mathrm{Ga}_{0.85} \mathrm{~N}(1.5 \mathrm{~nm} / 3.5 \mathrm{~nm})$ QWs sandwiched between a 400 -nm-thick $\mathrm{Al}_{0.82} \operatorname{In}_{0.18} \mathrm{~N}$ bottom cladding latticematched (LM) to $\mathrm{GaN}$ and a 100 -nm-thick $\mathrm{SiO}_{2}$ top cladding. It supports guided polariton eigenmodes arising from the strong coupling between GaN/AlGaN MQW excitons, of large binding energy $(\geqslant 40 \mathrm{meV})$ and oscillator strength $\left(\approx 1.5 \times 10^{13} \mathrm{~cm}^{-2}\right)[23,39]$, and the lowest order transverse electric $\left(\mathrm{TE}_{0}\right)$ guided mode. Metal gratings with an outcoupling length of $20 \mu \mathrm{m}$ were fabricated on the WGs to couple the propagating polaritons to free-space (Fig. 1). More details can be found in Appendix A. The use of an AlInN bottom cladding $\mathrm{LM}$ to $\mathrm{GaN}$ enables the growth of slab WGs that benefit from an excellent material quality, namely a reduced threading dislocation density leading to reduced interface

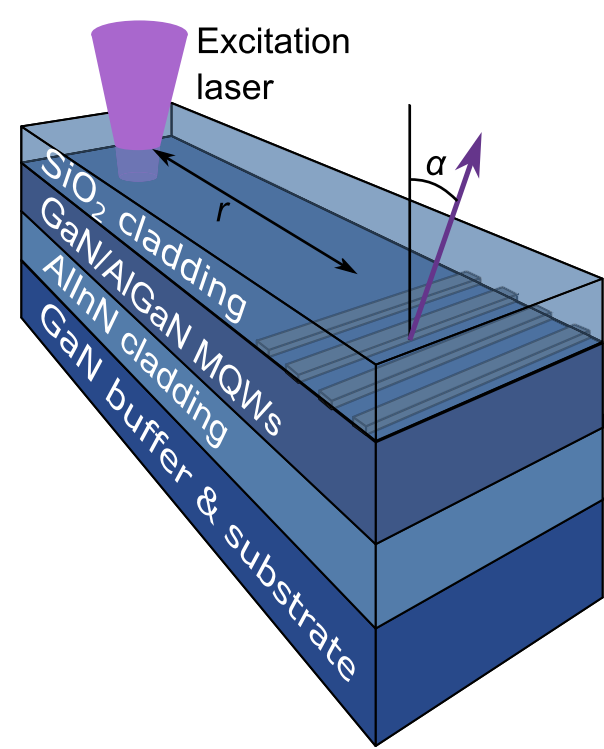

FIG. 1. Sketch of the sample structure and the associated experimental geometry.

roughness for both the cladding/WG and the $\mathrm{QW} /$ barrier regions $[23,40]$.

In order to uncover the relaxation dynamics of guided polaritons, the dispersion of the guided modes was measured under cw excitation by means of Fourier-space spectroscopy using real-space filtering to select the light outcoupled by a given grating. A representative parameter space, which consists of the propagation distance (i.e., the distance between the excitation spot and the collection grating), the sample temperature, and the excitation power density, was investigated to better understand guided polariton relaxation effects in these structures. The experimental setup is similar to the one described in Ref. [23]. A cw frequency-doubled optically pumped semiconductor laser emitting at $244 \mathrm{~nm}$ and the forth harmonic of a cw neodymium-doped yttrium aluminum garnet laser emitting at $266 \mathrm{~nm}$ were used for the excitation, the latter being used for the power series related to polariton nonlinearities to increase the injection efficiency and minimize any potential sample damage. The laser beam was coupled into an $80 \times$ UV microscope objective-with 0.55 numerical aperture and a $350 \mu \mathrm{m}$ field of view-through a so-called $4 f$ configuration of two lenses and a mirror. The laser spot diameter on the sample was about $10 \mu \mathrm{m}$ with the $244 \mathrm{~nm}$ laser and $20 \mu \mathrm{m}$ with the $266 \mathrm{~nm}$ laser, the latter spot size being more favorable for the development of polaritonrelated nonlinearities. This configuration allows us to scan the excitation spot over the sample within the field of view of the objective in two dimensions by rotating the mirror. The light emitted by the sample was then collected through the same objective lens and the back focal plane (Fourier plane) was imaged onto the spectrometer entrance slit by two lenses. The spectrometer consists of a liquid-nitrogen-cooled back-illuminated UV-enhanced charge-coupled device (CCD) mounted on a 55-cm-focal-length monochromator. A pinhole was placed in the first real-space image plane of the sample to select only the light emitted from the grating outcoupler. The pinhole diameter of $1.5 \mathrm{~mm}$ corresponds to a $20 \mu \mathrm{m}$ diameter 

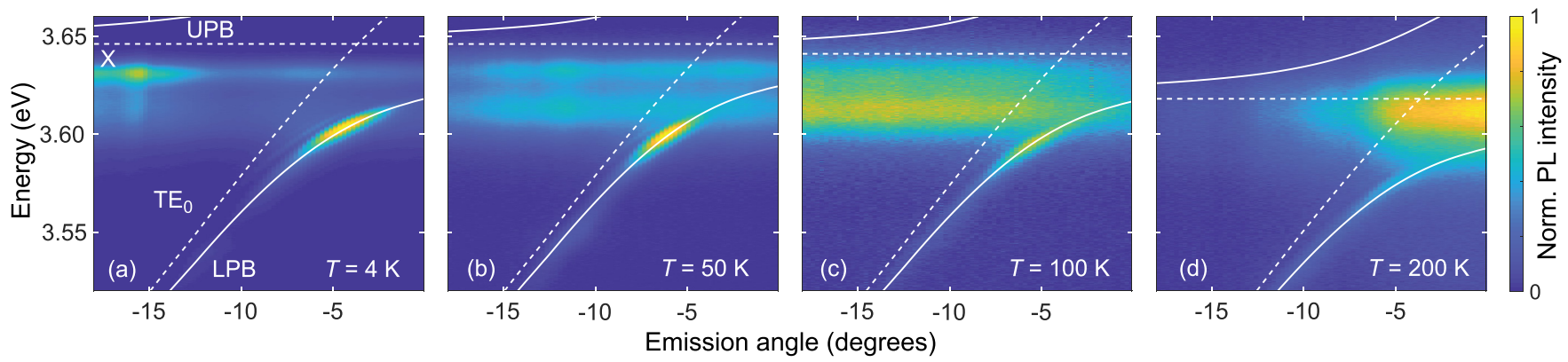

FIG. 2. Experimental dispersions of propagating LPs collected via metal grating outcouplers using cw $244 \mathrm{~nm}$ excitation with a $10 \mu \mathrm{m}$ spot diameter and $8.3 \mathrm{~kW} / \mathrm{cm}^{2}$ excitation power density. The measurements were taken at (a) $4 \mathrm{~K}$ and a propagation distance of $53 \mu \mathrm{m}$, (b) $50 \mathrm{~K}$ and $45 \mu \mathrm{m}$, (c) $100 \mathrm{~K}$ and $49 \mu \mathrm{m}$, and (d) $200 \mathrm{~K}$ and $28 \mu \mathrm{m}$, respectively. The fitted upper and lower polariton branches accounting for refractive index dispersion are shown by continuous lines and uncoupled modes $\left(X\right.$ and $\left.\mathrm{TE}_{0}\right)$ by dashed lines.

circle on the sample for the lens combination in use. A wire grid polarizer with an extinction coefficient better than 100:1 between 300 and $400 \mathrm{~nm}$ was positioned immediately after the pinhole to perform polarization-dependent measurements. An additional lens was flipped into the collection beam path to image the real space of the sample onto the spectrometer CCD while positioning the sample and the laser excitation spot. A blue light-emitting diode was used for illumination. The sample was mounted in a cold-finger continuous-flow liquid-helium cryostat.

Let us note that the largest excitation power density achieved with the $266 \mathrm{~nm}$ laser $\left(8000 \mathrm{~W} / \mathrm{cm}^{2}\right)$ leads to an overall injected carrier density equal to $3.2 \times 10^{12} \mathrm{~cm}^{-2}$, i.e., an average carrier density of $\sim 1.5 \times 10^{11} \mathrm{~cm}^{-2}$ per QW. Assuming as a first approximation that the carriers are photogenerated where light absorption takes place, one would expect a carrier density about $3 \times 10^{11} \mathrm{~cm}^{-2}$ in the first QW while it would decrease by about one order of magnitude and amount to $2 \times 10^{10} \mathrm{~cm}^{-2}$ when reaching the last QW. Such an injection profile has been previously reported in similar $\mathrm{GaN} / \mathrm{AlGaN}$ multiple QWs [40]. Those values indicate that the relevant carrier densities lie well below that leading to the Mott transition [41] and that the strong coupling regime is preserved at all times in the following experiments.

\section{RESULTS}

\section{A. Polariton relaxation}

Figure 2 shows four dispersion curves recorded at different temperatures after a propagation distance $(r)$ of around $50 \mu \mathrm{m}$ (except for data recorded at $200 \mathrm{~K}$, where the signal was too weak to be measured at such a distance) using an $8.3 \mathrm{~kW} / \mathrm{cm}^{2}$ excitation power density, which was the maximum available to us, at an excitation wavelength of $244 \mathrm{~nm}$ (cf. Appendixes $\mathrm{B}$ and $\mathrm{C}$ for details about the analysis of those dispersions). We observe that the LP branch (LPB) intensity decreases with temperature relative to the nondispersive photoluminescence (PL) background, making it impossible to observe the LPB above $200 \mathrm{~K}$. Note that as usual with wide band gap semiconductors, the upper polariton branch (UPB) is absent in these structures under nonresonant excitation $[23,42]$. We also observe that the polariton PL peak relaxes toward lower energy states along the branch and broadens with increasing temperature. This is the main signature of polariton relaxation that we study in this paper.

The effect of relaxation becomes even clearer when considering the relative LPB population $\left(N_{\text {pol }}\right)$ shown in Fig. 3(a). To obtain a reliable determination of the LPB population, special care was taken to subtract the cross-polarized PL signal, i.e., the polarization parallel to the propagation direction, from the TE-polarized signal in order to reduce the unpolarized PL background, which is not of polaritonic nature (Appendix D).
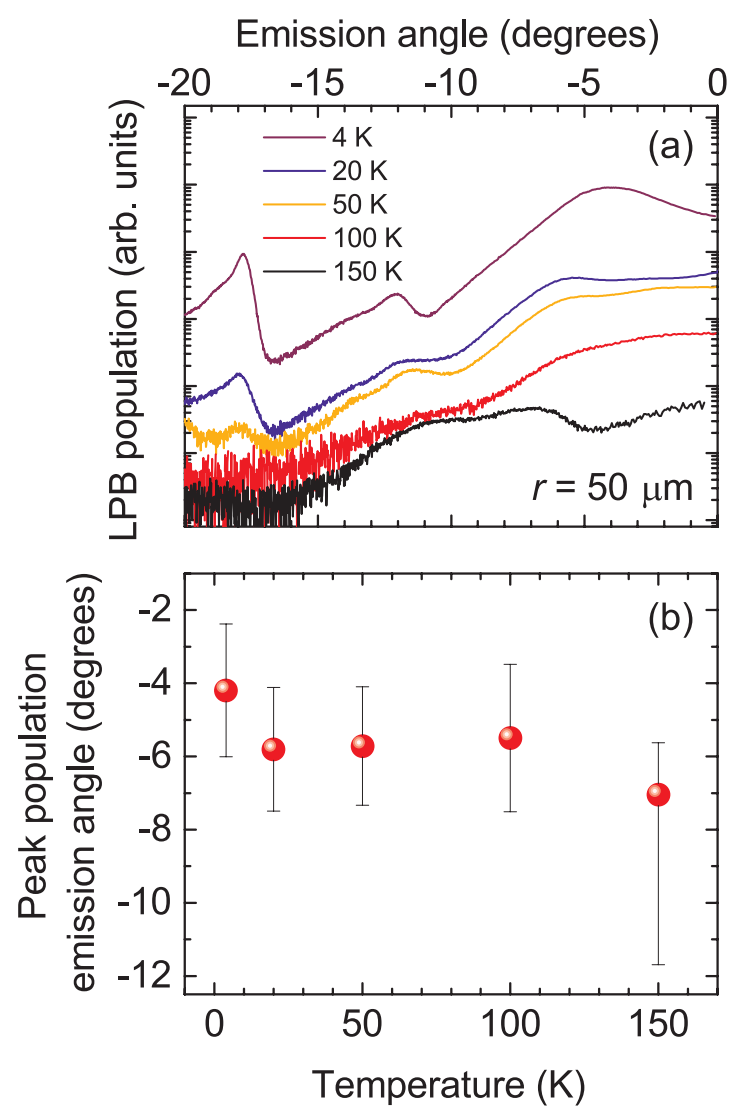

FIG. 3. LP relaxation as a function of temperature for a propagation distance of $50 \mu \mathrm{m}$ recorded at a power density of $8.3 \mathrm{~kW} / \mathrm{cm}^{2}$ using cw $244 \mathrm{~nm}$ excitation. (a) Relative LPB population for different temperatures. (b) LP peak emission angle (red dots) of the population shown in (a). The error bars show the FWHM of the population peak including the signature of an eventual asymmetry. 
In practice, the LP intensity $\left(I_{\mathrm{LP}}\right)$ was integrated over energy for each emission angle $\alpha$ from $E_{\mathrm{LPB}}(\alpha)-\Delta E(\alpha)$ to $E_{\mathrm{LPB}}(\alpha)+\Delta E(\alpha)$, with $\Delta E(\alpha)$ the full width at half maximum (FWHM) of the polariton emission for any given emission angle. Finally, $N_{\text {pol }}$ was calculated using the relation$\operatorname{ship} N_{\mathrm{pol}}=I_{\mathrm{LP}} \tau_{\mathrm{pol}}$, with $\tau_{\mathrm{pol}}$ the polariton lifetime determined as described in Appendix E. Even though this signal still contains artifacts from dispersionless PL contributions stemming from the QW longitudinal optical (LO) phonon replica $\left(\alpha \sim-12^{\circ}\right)$ and substrate luminescence $\left(\alpha \sim-18^{\circ}\right)$, the latter behave as spurious signals occurring at specific energies whose contribution can be safely ignored when comparing experimental data to numerical simulations (see below). The angle $\alpha$ corresponding to the position of the peak in the LPB population is plotted in Fig. 3(b) as a function of temperature. An overall decrease in the emission angle with temperature can be observed, which is an evidence of the enhancement of polariton relaxation at higher temperatures. However, the change in the angle remains small and the behavior of the population maximum can be considered as relatively robust.

To describe theoretically the polariton relaxation that occurs during the propagation of the guided modes, we use the semiclassical Boltzmann equations with spatial resolution [21], according to the general procedure described in Refs. [43,44] for each spatial cell and taking into account the transfer between these cells with a group velocity $v_{g}=\partial \omega / \partial k$ determined by the dispersion with $\omega$ the angular frequency. The size of the spatial cells is chosen to be $10 \mu \mathrm{m}$, a compromise between the numerical costs and spatial precision. The accuracy of the numerical simulations is thus comparable to the resolution of the experimental measurements (see, e.g., Fig. 6 hereafter). At this scale, the distribution function exhibits smooth variations, at least for sufficiently low densities. The equations read

$$
\begin{aligned}
\frac{\partial n_{k, r}}{\partial t}= & P_{\boldsymbol{k}, r}-\Gamma_{\boldsymbol{k}} n_{\boldsymbol{k}, r}-n_{\boldsymbol{k}, r} \sum_{\boldsymbol{k}^{\prime}} W_{\boldsymbol{k} \rightarrow \boldsymbol{k}^{\prime}, r}\left(n_{\boldsymbol{k}^{\prime}, r}+1\right) \\
& +\left(n_{\boldsymbol{k}, r}+1\right) \sum_{\boldsymbol{k}^{\prime}} W_{\boldsymbol{k}^{\prime} \rightarrow \boldsymbol{k}, r} n_{\boldsymbol{k}^{\prime}, r}-v_{g}(k) \frac{\partial n_{\boldsymbol{k}, r}}{\partial r} .
\end{aligned}
$$

Here $n_{k, r}$ is the polariton distribution function within a spatial cell located at position $r$. It describes the population both in the polaritonic part of the dispersion and in the excitonic reservoir, depending on the wave vector $\boldsymbol{k}$. The decay rate $\Gamma_{\boldsymbol{k}}$ includes both the nonradiative exciton decay and the photonic decay discussed in Appendix E. The pumping term $P_{k, r}=P_{0} \exp \left[-\left(E_{k}-E_{X}\right) / k_{B} T\right] \delta(r)$ - where $P_{0}$ is a prefactor, $E_{k}$ is the energy along the lower polariton branch, and $E_{X}$ is the uncoupled exciton energy at the center of the first Brillouin zone-describes a spatially localized pumping acting only within a single spatial cell and within the excitonic reservoir $\left(E_{k}>E_{X}\right)$, which corresponds to the rapid formation of thermalized excitons from the initially injected electrons and holes. The scattering rates $W_{\boldsymbol{k} \rightarrow \boldsymbol{k}^{\prime}, r}$ include both excitonphonon and exciton-exciton scattering. They depend on the density and distribution of particles within each spatial cell, which means that the linear increase in the area of the cells with propagation distance due to the radial outspread of the waveguided polaritons is accounted for [23].
A typical example of the results of the simulations is shown in Fig. 4, which presents the polariton distribution function $n_{k, r}$ at $150 \mathrm{~K}$, plotted as a function of relative energy $E_{k}(=E-$ $\left.E_{X}\right)$ for two different positions $r$ : Below the pump $(r=0$, red dashed line) and far from the pump $(r=50 \mu \mathrm{m}$, black solid line). The experimental distribution function measured at $150 \mathrm{~K}$ at $r=50 \mu \mathrm{m}$ from the excitation spot is shown as a blue solid line. The same experimental data are plotted in Fig. 3(a) as a function of detection angle, with the link between the energy and the angle given by the dispersion curve shown in Fig. 2. Below the pump, the population bottleneck occurs just below the exciton energy. During their free propagation, polaritons redistribute themselves differently along the dispersion curve. The excitons injected by the pump do not propagate outside the pumping spot, because their group velocity is small. In other words, the diffusion coefficient of free excitons is negligible because of their large effective mass. Due to the low population of excitonic states, thermal processes lead to a rapid transfer of the polaritons from the upper part of the bottleneck region toward higher energies, where the excitonic states are relatively empty. Indeed, their lifetime is short compared to the pure photonic states of the guided modes because of the emission into nonpolariton states, as observed in Fig. 2. This leads to a rapid decrease of the polariton population at energies just below $E_{X}$. At the same time, the main part of the overall population continues to relax downward. Because of both processes, the maximum of the distribution function shifts toward lower energies during the propagation.

All relaxation processes are more efficient at higher temperatures, and therefore for a constant propagation distance this maximum is observed at lower energies for a higher temperature. The results of the simulations and experimental measurements at different temperatures are summarized in Fig. 5. We now focus on the relative energy $E-E_{X}$ of the LPB population maximum, and not on the angle $\alpha$. Experimental points [the same as those shown in Fig. 3(b)] are plotted here as red circles, and the theoretical values are plotted as black squares. A good quantitative agreement between the two data sets can be observed. While a clear overall decrease in the relative position of the population maximum with temperature can indeed be observed, we note that this position

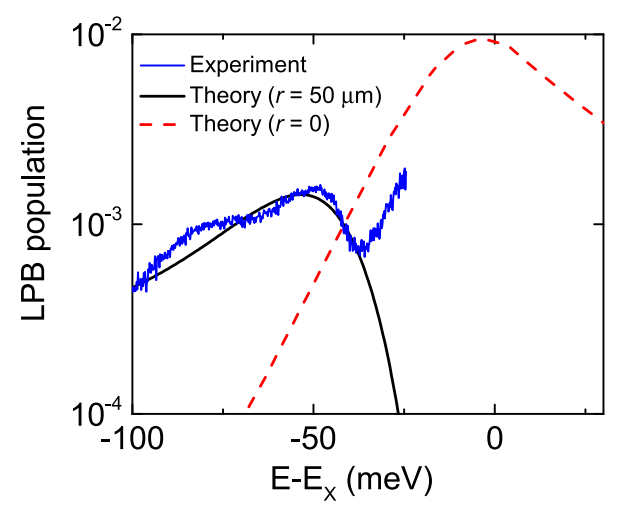

FIG. 4. LPB population at $T=150 \mathrm{~K}$ as a function of the relative energy $E-E_{X}$ : Simulations ( $r=0$, red dashed line; $r=50 \mu \mathrm{m}$, black solid line) and experiment ( $r=50 \mu \mathrm{m}$, blue line). 
can be considered as being relatively robust since it exhibits a small decrease over a substantial range of temperatures. The temperature dependence of the absolute emission energy of the LPB population maximum will obviously be a trade-off between the redshift of the free exciton, the position of the bottleneck shown in Fig. 4, and the decrease in the normal mode splitting (Appendix C). In particular, the shift in the exciton energy with increasing temperature is partly compensated by the decrease in the normal mode splitting leading to a reduced drift of the LPB population maximum with temperature. This property could prove especially useful for future devices based on guided polaritons, such as WG polariton lasers and amplifiers, as it would ensure a good stability of the operating wavelength compared to their planar microcavity counterparts where the energy position of the cavity mode adds to the complexity of this platform. In this respect, let us note that future WG structures could consist of a ridge-WG geometry in order to keep a sufficiently large polariton density for a given propagation distance, hence enabling to probe the stability of the bottleneck region up to room temperature.

To further quantify the polariton relaxation process, we have experimentally determined the evolution of both the position of the LP population maximum [Fig. 6(a)] and the total LP intensity [Fig. 6(b)] as a function of temperature and propagation distance $r$. As shown in Fig. 6(a), the peak in LP population progressively shifts to lower energies. This is due to the progressive polariton relaxation occurring during propagation and the increased absorption experienced by the higher-energy polaritons, which are closer to the QW band edge. This behavior is well reproduced by the theory accounting for the experimental detection conditions (solid lines). It is in agreement with the overall energy relaxation picture, illustrated by the theoretical predictions shown in Fig. 4 for $T=150 \mathrm{~K}$ (red and black lines for different spatial positions). Similarly, the total LP intensity decreases with increasing distance $r$ [Fig. 6(b)]. Note, however, that even though a linear decrease in the total LP intensity as function of $r$ is observed when using a semi-logarithmic scale (see, e.g., data recorded at $T=4 \mathrm{~K}$ ), such a behavior cannot be linked in a straightforward manner to the absorption coefficient as one could $a$ priori expect. Indeed, the LP emission covers a broad spectral range that coincides with the absorption tail of the WG, which prevents any straightforward theoretical determination of the

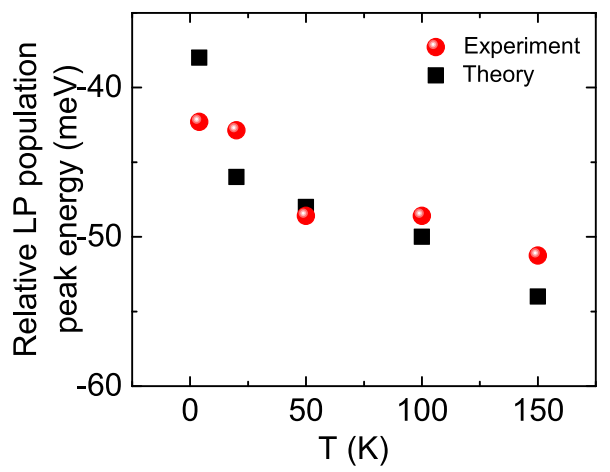

FIG. 5. Relative LPB population peak energy as a function of temperature for a propagation distance of $50 \mu \mathrm{m}$ : simulations (black squares) and experiments (red circles).
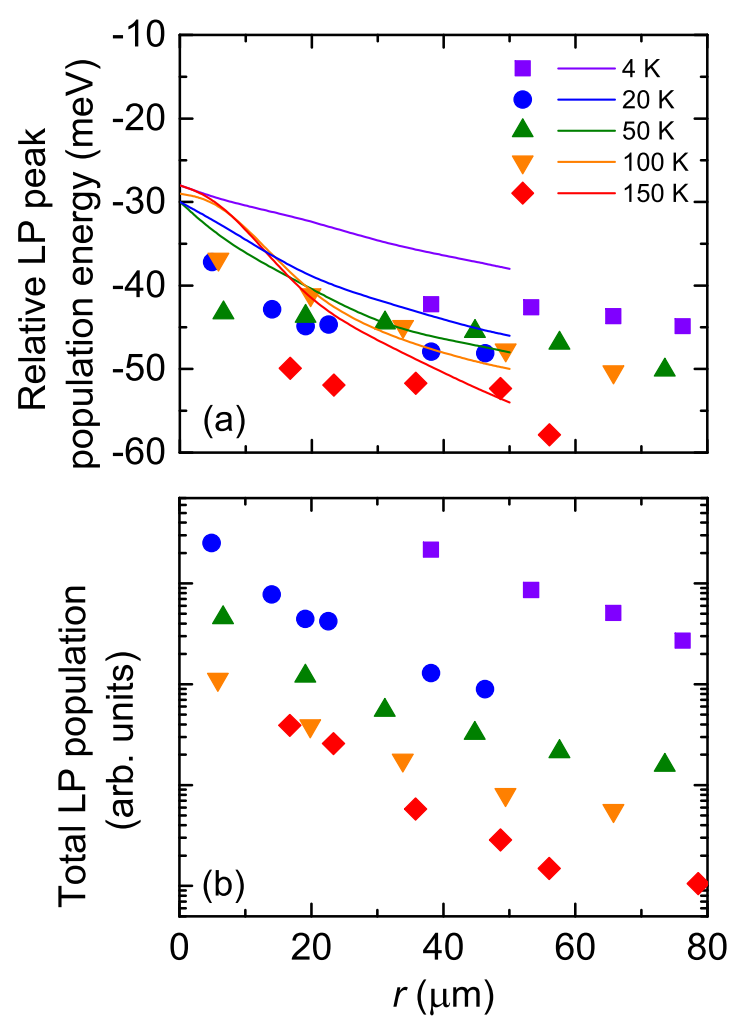

FIG. 6. (a) Change in the relative LP peak population energy, and (b) total LP intensity both as a function of propagation distance $r$ and recorded at a power density of $8.3 \mathrm{~kW} / \mathrm{cm}^{2}$ using cw $244 \mathrm{~nm}$ excitation at several temperatures. The symbols show the measured data, while the solid lines show the calculated values.

absorption coefficient. In addition, the mean absorption coefficient $\langle\alpha\rangle$ deduced from the data taken at $T=4 \mathrm{~K}$ amounts to $\sim 830 \mathrm{~cm}^{-1}$, a value most likely overestimated compared with the value of the absorption coefficient we reported previously on similar WG structures that essentially differed by their lower $\mathrm{Al}$ content in the $\mathrm{AlGaN}$ waveguide $\left(\alpha \sim 60 \mathrm{~cm}^{-1}\right.$ at around $200 \mathrm{meV}$ below the MQW free exciton energy in a WG with an $\mathrm{Al}$ content of 10\%) [23]. The fact that the picture cannot be reduced to energy-dependent absorption is confirmed by direct experimental measurements at $T=150 \mathrm{~K}$ (Fig. 16), showing an increase in the emission intensity at lower energies with increasing propagation distance. We can therefore conclude that the most accurate description of the processes is given by the Boltzmann equations (1), which include both relaxation and decay (with energy dependence), together with the propagation.

\section{B. Polariton nonlinearities}

Beyond the above-mentioned LP relaxation features, let us emphasize that at the lowest cryogenic temperature of $T=4 \mathrm{~K}$ investigated in this work, we observe the clear signature of a nonlinear polariton emission process that manifests itself through a nonlinear increase in LP population with increasing pump power density. The intensity integrated between $-9^{\circ}$ and $-1^{\circ}$ is rising with a power-law exponent of 2.7 between 640 and $1300 \mathrm{~W} / \mathrm{cm}^{2}$ as shown in Figs. 7(a) and 7(b). This increase slightly exceeds a quadratic 


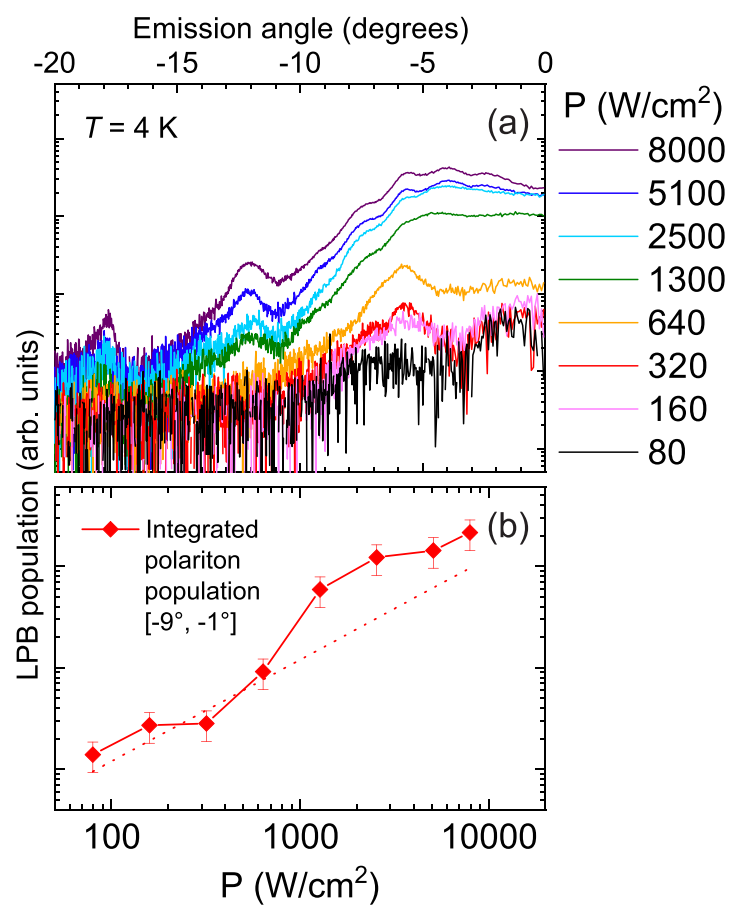

FIG. 7. LP population integrated over energy and recorded at different excitation power densities spanning two orders of magnitude using cw $266 \mathrm{~nm}$ excitation: (a) $N_{\mathrm{pol}}$ as a function of emission angle, and (b) as a function of excitation power density integrated over emission angles between $-9^{\circ}$ and $-1^{\circ}$. The dotted line corresponds to a linear increase in polariton population and serves as a guide to the eye.

dependence, which suggests that stimulated processes populating the modes take place rather than phonon- and/or exciton-assisted relaxation, since the latter would result in a quadratic population increase at most [45]. We have also mentioned that the carrier density per well does not exceed $3 \times 10^{11} \mathrm{~cm}^{-2}$ at the maximum power density value of 8000 $\mathrm{W} / \mathrm{cm}^{2}$ used in this work, which remains well in the range where excitonic effects dominate for this type of QWs [41]. This nonlinear increase in the LP emission is accompanied by a redistribution in $k$ space and the onset of several maxima that belong to the LP dispersion as can be seen in Fig. 8 .
In previous experiments involving polariton WGs [24], such modulation was associated with the Fabry-Perot modes of a horizontal cavity formed by regularly spaced cracks. Here the modulations we observe show a spacing in $k$ space that would be compatible with a cavity length of around $10 \mu \mathrm{m}$. In the absence of any horizontal cracks in those nearly LM structures, such an explanation is unlikely. Instead, such a feature suggests that those maxima could stem from a nonlinear process where the polariton modes first around $-6^{\circ}$, i.e., at the bottleneck, then at $\sim-8^{\circ}$ and at $\sim-4^{\circ}$ at higher pumping power densities get amplified [Fig. 7(a)]. LPs redistribute themselves along the dispersion curve through various mechanisms, including polariton-polariton scattering. Indeed, as discussed hereafter, a mode with substantial intensity, i.e., polariton occupation, is expected to be unstable against nonlinear parametric processes in a system characterized by a negative curvature of the dispersion, and in the present case, repulsive interaction between particles.

Let us note that there is no manifestation of such nonlinearities at $T=20 \mathrm{~K}$ and above (relevant measurements are shown in Appendix F), as the reduced phonon population generates the most favorable conditions for the onset of this type of nonlinearity. At higher temperatures, the required power density to reach this regime is likely beyond what can be achieved with the present setup.

The development of the optical parametric oscillator (OPO)-like process can be described theoretically by calculating the dispersion of the weak excitations of a macroscopically occupied mode located at the position of the highest population at a weaker power density. The imaginary part of the weak excitations determines their decay or growth rates, and the mode with the highest growth rate is the one where the OPO signal is expected to be detected. We begin with the GPE [46]:

$$
i \hbar \frac{\partial \psi}{\partial t}=\hat{H}_{0} \psi+g|\psi|^{2} \psi-\mu \psi,
$$

written for the order parameter $\psi$ of the macroscopically occupied state from which the OPO can occur. Here $\hat{H}_{0}$ is the Hamiltonian of the noninteracting system such that $\hat{H}_{0} \psi(k)=E(k) \psi(k), g$ is the coupling constant accounting for the strength of polariton-polariton interactions, and $\mu$ is the chemical potential that corresponds to the energy of the
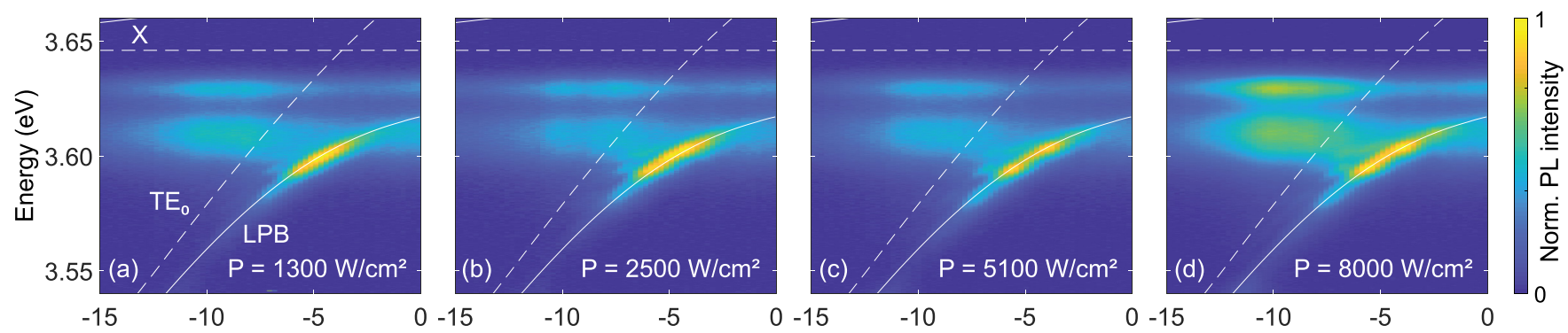

Emission angle (degrees)

FIG. 8. Measured dispersions of propagating LPs collected via metal grating outcouplers using cw $266 \mathrm{~nm}$ excitation with a $20 \mu \mathrm{m}$ spot diameter at a temperature of $4 \mathrm{~K}$ for increasing pump power densities. The measurements were taken at (a) 1300, (b) 2500, (c) 5100, and (d) $8000 \mathrm{~W} / \mathrm{cm}^{2}$, respectively. The fitted LPB accounting for refractive index dispersion is shown by a continuous line in each case together with uncoupled modes ( $X$ and $\mathrm{TE}_{0}$, dashed lines). 
macro-occupied mode with the interactions. Equation (2) is then diagonalized, and the dispersion of the weak excita-

$$
\operatorname{det}\left(\begin{array}{c}
E\left(k_{0}+k\right)-E\left(k_{0}\right)+g n-\hbar \omega \\
g n
\end{array}\right.
$$

where $n$ is the density of the macro-occupied mode, $k_{0}$ is its wave vector, and $k$ is the wave vector of the weak excitations (density waves in the condensate, also called "bogolons" [47]) measured relative to $k_{0}$. The dispersion being known, the only adjustable parameter is the interaction energy gn. It controls the value of the positive imaginary part of the bogolon energy, which should exceed the mode losses. Taking the best lifetime, obtained at $4 \mathrm{~K}$ for a detuning of $-40 \mathrm{meV}$ (see Fig. 14), gives an estimate of the mode linewidth of $0.5 \mathrm{meV}$. The $g n$ value also controls the wave vector and the energy at which the imaginary part becomes the largest, which indicates where the OPO should switch on first. The result of the calculations, using $g n=0.9 \mathrm{meV}$, is plotted in Fig. 9(a) over a relatively narrow range of in-plane wave vector values around the position of the bottleneck at lower pumping densities $k_{0} \approx 46.5 \mu \mathrm{m}^{-1}$. The position of the maxima of the imag-

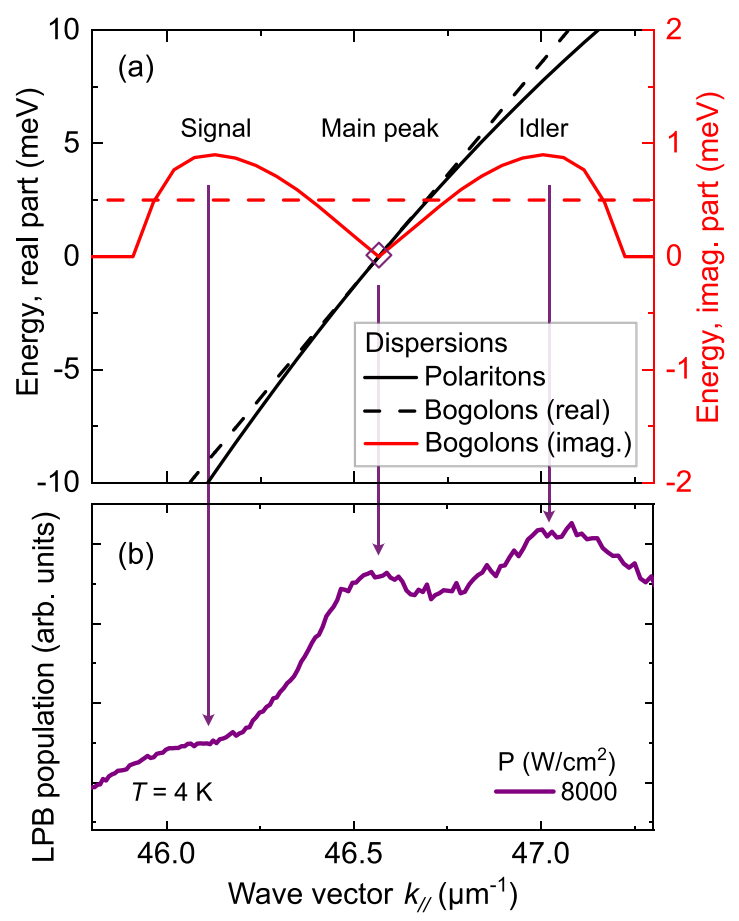

FIG. 9. (a) Theoretical calculation of the mode instabilities at $4 \mathrm{~K}$ : Bare polariton dispersion (black solid line), dispersion of weak excitations (bogolons, real part-black dashed line, imaginary part—red solid line). The horizontal dashed line shows the minimal estimated value of losses. (b) Close-up view of the lower polariton population as a function of in-plane wave vector measured at the highest power density of $8000 \mathrm{~W} / \mathrm{cm}^{2}$ in the vicinity of the bottleneck [cf. Fig. 7(a)]. The vertical arrows connect the position of the maxima of the imaginary part where the OPO (main peak as well as the signal and idler peaks) is expected to appear and their signature in the experimental spectrum. tions $\hbar \omega(k)$ is given by solving the following characteristic equation:

$\left.\begin{array}{c}g n \\ E\left(k_{0}-k\right)-E\left(k_{0}\right)+g n+\hbar \omega\end{array}\right)=0$,

inary part of the energy of the weak excitations $\operatorname{Im}(\hbar \omega(k))$ is shown by a red solid line, which reproduces well the experimentally observed position of the maxima of the OPO emission shown in Fig. 9(b). The real part of the dispersion of the weak excitations $\operatorname{Re}[\hbar \omega(k)]$ (black dashed line) shows a relatively small renormalization with respect to the bare polariton dispersion $E(k)$ (black solid line), which is also in agreement with the experiment. Hence, the OPO mechanism appears as the most plausible explanation accounting for our experimental data, especially when considering its sensitivity against an increase in lattice temperature. We also want to point out that the amplification and the nonlinear effect we observe occur in the absence of any optical feedback. It explains why the intensity rise remains moderate and why the modulations in the emission are quite broad. In order to properly harness those nonlinearities, an appropriate cavity design such as a ridge WG cavity or a microring resonator geometry should be implemented. Finally, let us note that the observation of parametric instabilities in such a WG structure does correspond to a specific type of polariton-driven optical nonlinearities. Indeed, using different pumping conditions, e.g., under pulsed excitation, the balance between gain and nonlinear losses may also lead to the generation of solitonic features [35,36]. Thus, self-phase modulation, a precursor stage for the formation of optical solitons has recently been reported under resonant pulsed excitation up to room temperature in similar GaN/AlGaN WGs [48].

\section{CONCLUSION}

In conclusion, we have studied polariton relaxation along the lower polariton branch in strongly coupled GaN/AlGaN WGs both theoretically and experimentally. We have observed that the energy position of the polariton emission maximum is relatively robust versus temperature due to a balance between the shift of the exciton energy and the change in the normal mode splitting, a promising feature when aiming for applications such as lasers and amplifiers. A four-wave mixing OPO signal, which is a signature of nonlinear polariton interactions, is observed at high pumping at $4 \mathrm{~K}$ in the $\mathrm{cw}$ regime. Experimental results and simulations are in good quantitative agreement, in particular theoretical calculation of the mode instabilities at $4 \mathrm{~K}$ match with the experimental OPO process when considering an interaction energy $g n=0.9 \mathrm{meV}$ for polariton nonlinearities.

\section{ACKNOWLEDGMENTS}

We would like to thank the Swiss National Science Foundation for financial support through Grant No. 200020_162657. We acknowledge the support of the project "Quantum Fluids of Light" (ANR-16-CE30-0021) and that of the ANR 


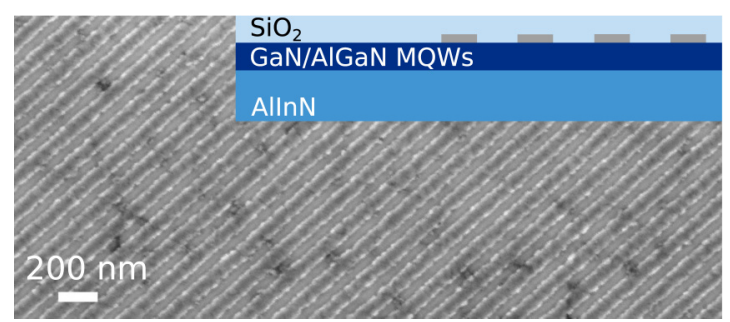

FIG. 10. SEM image of a representative evaporated Al grating after lift-off and before $\mathrm{SiO}_{2}$ deposition. Inset: Sketch of the sample structure. Gratings extend over $100 \times 100 \mu \mathrm{m}^{2}$ areas and have a $130 \mathrm{~nm}$ period. The $\mathrm{Al}$ stripes are $70 \mathrm{~nm}$ wide.

program "Investissements d'Avenir" through the IDEX-ISITE initiative 16-IDEX-0001 (CAP 20-25) and GANEXT (ANR11-LABX-004). We also wish to thank the support of the Center of MicroNanotechnology (CMi) at EPFL and its technical staff.

\section{APPENDIX A: SAMPLE DETAILS}

The structure under investigation is similar to the one used in our previous work on polariton WGs [23] and consists of a 130-nm-thick active region with 22 embedded $\mathrm{GaN} / \mathrm{Al}_{0.15} \mathrm{Ga}_{0.85} \mathrm{~N}(1.5 \mathrm{~nm} / 3.5 \mathrm{~nm})$ QWs sandwiched between a 400 -nm-thick $\mathrm{Al}_{0.82} \mathrm{In}_{0.18} \mathrm{~N}$ bottom cladding lattice matched to $\mathrm{GaN}$ and a 100 -nm-thick $\mathrm{SiO}_{2}$ top cladding. The bottom cladding and the active region were grown by metalorganic vapor phase epitaxy in an AIXTRON 200/4 RF-S reactor on a low dislocation density $\left(\sim 10^{6} \mathrm{~cm}^{-2}\right) c$-plane FS GaN substrate. The AlInN bottom cladding layer contains seven 5-nm-thick GaN interlayers positioned $50 \mathrm{~nm}$ apart in order to avoid kinetic roughening of the AlInN alloy [49]. All aforementioned layer thicknesses were determined by highresolution $\mathrm{x}$-ray diffraction.

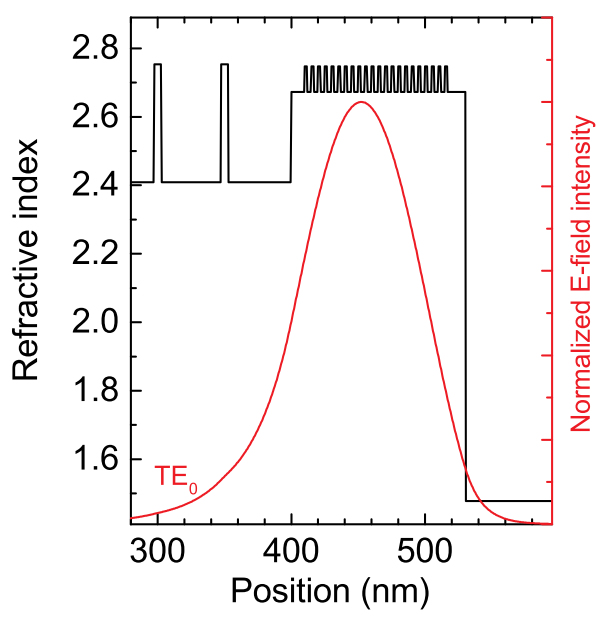

FIG. 11. Optical mode profile along the growth axis, calculated with a finite-difference time-domain mode solver, of the $\mathrm{TE}_{0}$ mode supported by the waveguide. The position is measured from the interface between the GaN buffer and the bottom cladding. The refractive index for (Al)GaN is taken from Ref. [51], while the refractive index of AlInN is taken from Ref. [52], and the refractive index of $\mathrm{SiO}_{2}$ is issued from experimental data.

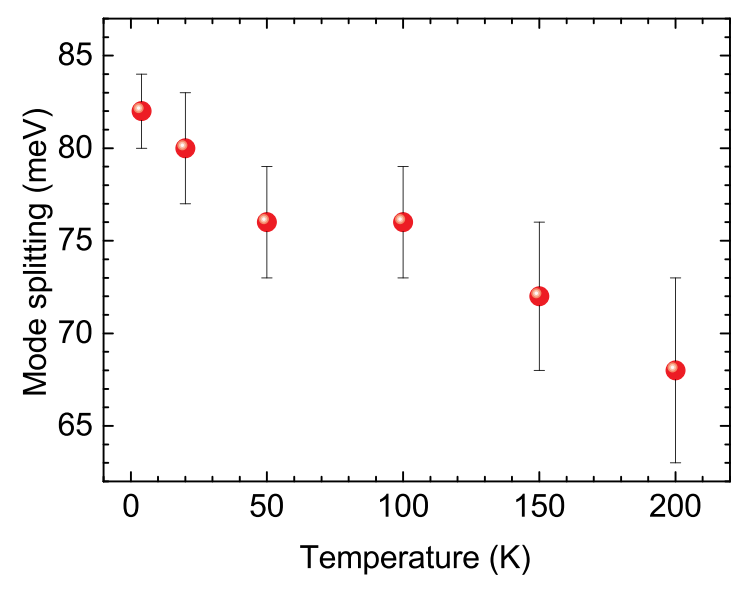

FIG. 12. Normal mode splitting derived from the fitted waveguide polariton dispersions as a function of temperature. The error bars correspond to the standard deviation of the mode splitting averaged over several measurements.

Metal grating outcouplers with a $130 \mathrm{~nm}$ period spanning $100 \times 100 \mu \mathrm{m}^{2}$ were fabricated on the WGs using a poly(methylmethacrylate) (PMMA) resist double layer. A PMMA 495 and a PMMA 950 layer were subsequently spin coated on the sample, and the grating pattern was written using a $100 \mathrm{keV}$ Vistec EBPG5000 e-beam lithography system. After resist development, a 2-nm-thick Ti film followed by a 20-nm-thick Al film were evaporated using a $1 \mathrm{~m}$ working distance e-beam evaporator. Lift-off of the metal was performed in remover 1165 at $75^{\circ} \mathrm{C}$. Figure 10 shows a scanning electron microscopy (SEM) image of a fabricated grating. Finally, a $\mathrm{SiO}_{2}$ top cladding was deposited by plasma-enhanced chemical vapor deposition on top of the active region, burying the gratings.

Figure 11 shows the refractive index profile of the waveguide along the normal to the sample surface, as well as the lowest order $\mathrm{TE}_{0}$ guided mode profile, which was calculated using a finite-difference time-domain solver [50]. The

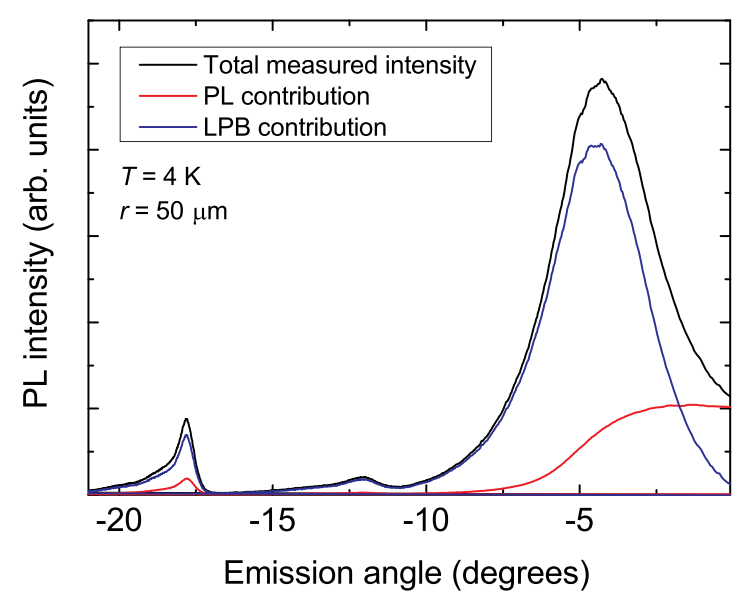

FIG. 13. Representative determination of the LPB PL signal (blue line) for a propagation distance $r=50 \mu \mathrm{m}$ via the subtraction of the cross-polarized excitonic PL background contribution (red line) from the total measured PL intensity (black line). 


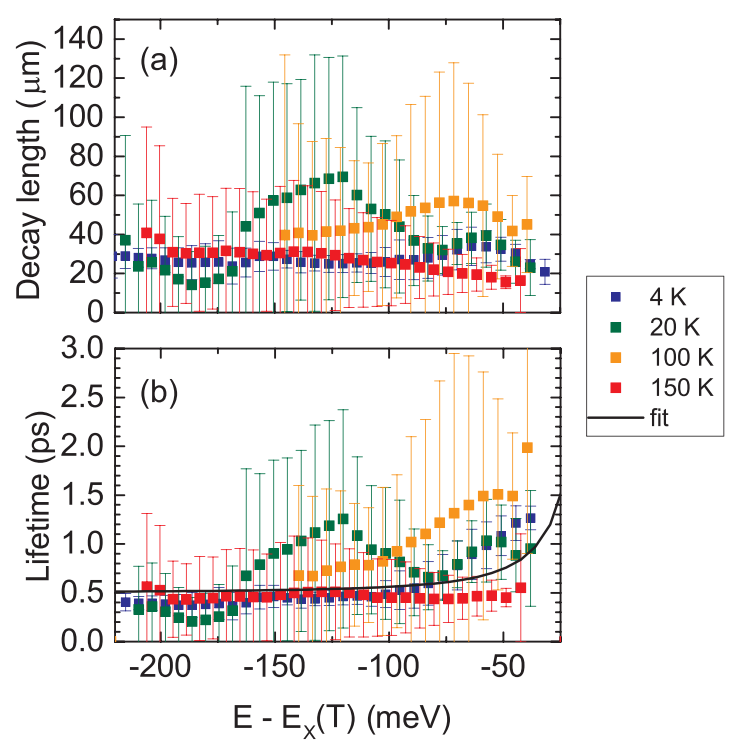

FIG. 14. (a) Experimental LP decay length as a function of polariton energy at different temperatures. (b) Deduced lifetime of the guided LPs (symbols). A fit to Eq. (E1), taking all the reported data into account, is shown with the continuous black line. Note that the data are plotted relative to the exciton energy, which is temperature dependent. The data corresponding to different temperatures are therefore directly comparable with each other. The error bars represent the $90 \%$ confidence interval of the exponential decay function that was fitted to the data.

effective length of the $\mathrm{TE}_{0}$ mode, defined as

$$
L_{\mathrm{eff}}=\frac{\int \varepsilon|E|^{2} d y}{\max \left(\varepsilon|E|^{2}\right)},
$$

with $\varepsilon$ the dielectric constant and $E$ the electric field, is $102 \mathrm{~nm}$ at a wavelength of $340 \mathrm{~nm}$.

\section{APPENDIX B: COUPLED OSCILLATOR MODEL}

In the coupled oscillator model (COM), the coupled photonic and excitonic states are described by the following Hamiltonian expressed in matrix form:

$$
\widehat{H}=\left[\begin{array}{cc}
E_{X}+i \gamma_{X} & g_{0} \\
g_{0} & E_{P}+i \gamma_{P}
\end{array}\right],
$$

where $E_{X}, \gamma_{X}$ and $E_{P}, \gamma_{P}$ are the energy and decay rate of the exciton and the photon, respectively, and $g_{0}$ is the coupling strength between both modes. $E_{X}$ was determined experimentally by photoluminescence measurements while accounting for exciton localization at low temperatures [23]. $E_{P}$ was determined as a function of the in-plane wave vector $\left(k_{/ /}\right)$and emission angle by finite-difference time-domain calculations, which take the refractive index dispersion of the materials into account. Let us note that in wide band gap semiconductors, ignoring the refractive index dispersion results in an overestimation of the normal mode splitting [23]. The eigenstates of the system are the UPB and LPB and can be found by diagonalizing this Hamiltonian:

$$
\begin{aligned}
E_{\mathrm{UPB}}= & \frac{1}{2}\left[E_{X}+E_{P}+i\left(\gamma_{X}+\gamma_{P}\right)\right] \\
& +\frac{1}{2} \sqrt{4 g_{0}^{2}-\left(\gamma_{X}-\gamma_{P}\right)^{2}+\left(E_{X}-E_{P}\right)^{2}}, \\
E_{\mathrm{LPB}}= & \frac{1}{2}\left[E_{X}+E_{P}+i\left(\gamma_{X}+\gamma_{P}\right)\right] \\
& -\frac{1}{2} \sqrt{4 g_{0}^{2}-\left(\gamma_{X}-\gamma_{P}\right)^{2}+\left(E_{X}-E_{P}\right)^{2}} .
\end{aligned}
$$

The normal mode splitting $\Omega_{\text {Rabi }}$ is defined at zero detuning $\left(E_{X}=E_{P}\right.$ at $\left.k_{/ /}=0\right)$. By subtracting the two energies, we obtain

$$
\Omega_{\text {Rabi }}=\sqrt{4 g_{0}^{2}-\left(\gamma_{X}-\gamma_{P}\right)^{2}} .
$$

Equation (B4) was fitted to the measured dispersion curves with $g_{0}$ as a free parameter.

\section{APPENDIX C: NORMAL MODE SPLITTING EVOLUTION}

The normal mode splitting was deduced from a fit of the COM described above, including refractive index dispersion of the materials. The splitting is shown in Fig. 12 and is larger compared to what was reported in our previous work [23], due to the larger exciton oscillator strength, which originates from the increased $\mathrm{Al}$ content in the QW barriers from $10 \%$ to $15 \%$. We observe a moderate decrease in the splitting with increasing temperature, which is a likely consequence of the increased homogeneous broadening of the exciton. We did not observe any significant variation in the normal mode splitting with propagation length or excitation power, even though both parameters influence the polariton density at the grating outcoupler.
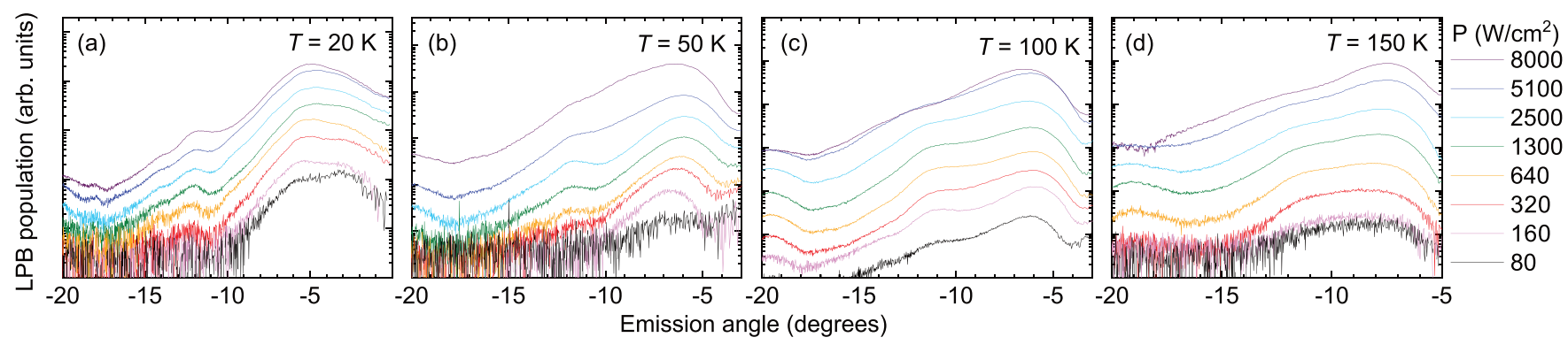

FIG. 15. LP population integrated over energy at different excitation power densities, spanning two orders of magnitude, and at different temperatures using cw $266 \mathrm{~nm}$ excitation with a $20 \mu \mathrm{m}$ spot diameter: (a) 20, (b) 50, (c) 100, and (d) $150 \mathrm{~K}$. 


\section{APPENDIX D: PHOTOLUMINESCENCE BACKGROUND SUBTRACTION}

The waveguide dispersion was measured as described above. A wire-grid polarizer was used to record both the light polarized along the TE direction, as well as the crosspolarized signal (with polarization parallel to propagation direction). The cross-polarized signal was subtracted from the TE-polarized signal in order to eliminate the PL background (unpolarized) from the waveguide emission (TE polarized). Despite this procedure, some dispersionless PL signal stemming from the QWs and the substrate is still visible, as can be seen in Fig. 2 of the main text. The remaining dispersionless PL signal in the polariton intensity was reduced by subtracting the integrated cross-polarized intensity multiplied by a normalization factor. An example of such a data treatment is shown in Fig. 13.

\section{APPENDIX E: POLARITON LIFETIME AND DECAY LENGTH}

The polariton decay length $d$ was determined by moving the excitation spot away from the grating. With increasing propagation distance $r$, the equation $\frac{A}{r} \exp (-r / d)$ was fitted to the measured intensity for each detuning with $d$ and the normalization coefficient $A$ used as fitting parameters. The $\frac{1}{r}$ dependence accounts for the radial polariton outspread, while the $\exp (-r / d)$ term describes the polariton decay. The polariton lifetime $\tau_{\text {pol }}$ was calculated as $\frac{d}{v_{g}}$, with the polariton group velocity $v_{g}$ determined from the LP dispersion. The polariton lifetime is the weighted average of the photon and exciton lifetimes $\tau_{p}$ and $\tau_{X}$, respectively:

$$
\frac{1}{\tau_{\mathrm{pol}}\left(k_{/ /}\right)}=\frac{\left|P\left(k_{/ /}\right)\right|^{2}}{\tau_{p}}+\frac{\left|X\left(k_{/ /}\right)\right|^{2}}{\tau_{X}}
$$

where $P$ and $X$ are the Hopfield coefficients with $\left|P\left(k_{/ /}\right)\right|^{2}$ and $\left|X\left(k_{/ /}\right)\right|^{2}$ giving the photon and exciton fraction of the polaritons, respectively [2]. The experimentally determined decay lengths and lifetimes are shown in Fig. 14 as a function of polariton energy.

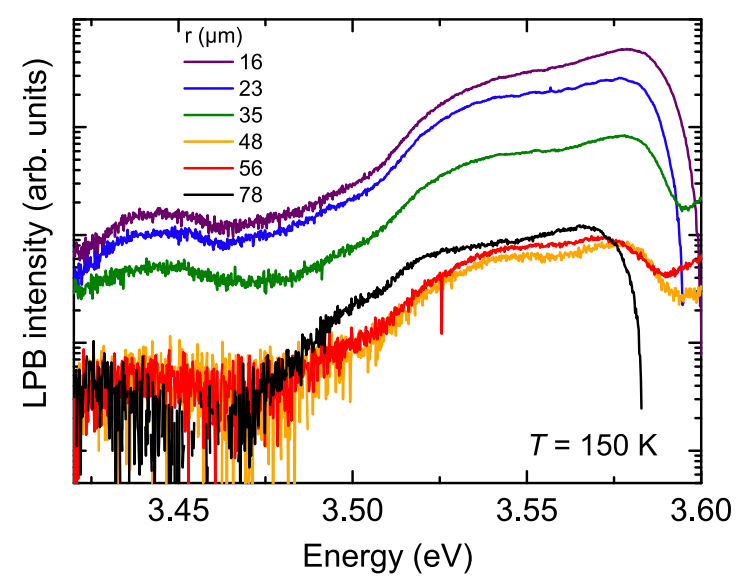

FIG. 16. LPB emission intensity spectrum as a function of energy at $T=150 \mathrm{~K}$ for different propagation distances $r$. The observed increase in the emission intensity around $E=3.55 \mathrm{eV}$ is due to the efficient polariton relaxation from higher energies.

\section{APPENDIX F: POWER-DEPENDENT POLARITON POPULATION}

In addition to the power-dependent polariton population measured at $4 \mathrm{~K}$ shown in Fig. 7 of the main text, the same experiment was also repeated at higher temperatures up to $150 \mathrm{~K}$. The results are shown in Fig. 15. Contrary to the behavior reported at $4 \mathrm{~K}$, no nonlinear increase in polariton population and no interference fringes are observed at higher temperatures.

\section{APPENDIX G: LOWER POLARITON EMISSION SPECTRA AT DIFFERENT DISTANCES}

In order to prove that our experimental observations cannot be reduced to simple energy-dependent absorption, we show an example of emission intensity spectra measured at $T=$ $150 \mathrm{~K}$ at different distances $r$ from the laser spot (Fig. 16). At this temperature, the relaxation is so efficient that it overcomes absorption at lower energies, and the emission intensity at these energies (around $E=3.55 \mathrm{eV}$ ) grows instead of decaying with the propagation distance. This clearly confirms that a correct model of the experimental system should clearly include energy relaxation.
[1] S. I. Pekar, Theory of electromagnetic waves in a crystal with excitons, J. Phys. Chem. Solids 5, 11 (1958).

[2] J. J. Hopfield, Theory of the contribution of excitons to the complex dielectric constant of crystals, Phys. Rev. 112, 1555 (1958).

[3] C. Weisbuch, M. Nishioka, A. Ishikawa, and Y. Arakawa, Observation of the Coupled Exciton-Photon Mode Splitting in a Semiconductor Quantum Microcavity, Phys. Rev. Lett. 69, 3314 (1992).

[4] P. M. Walker, L. Tinkler, M. Durska, D. M. Whittaker, I. J. Luxmoore, B. Royall, D. N. Krizhanovskii, M. S. Skolnick, I. Farrer, and D. A. Ritchie, Exciton polaritons in semiconductor waveguides, Appl. Phys. Lett. 102, 012109 (2013).
[5] A. Imamoglu, R. J. Ram, S. Pau, and Y. Yamamoto, Nonequilibrium condensates and lasers without inversion: Excitonpolariton lasers, Phys. Rev. A 53, 4250 (1996).

[6] P. G. Savvidis, J. J. Baumberg, R. M. Stevenson, M. S. Skolnick, D. M. Whittaker, and J. S. Roberts, Angle-Resonant Stimulated Polariton Amplifier, Phys. Rev. Lett. 84, 1547 (2000).

[7] M. Saba, C. Ciuti, J. Bloch, V. Thierry-Mieg, R. André, Le Si Dang, S. Kundermann, A. Mura, G. Bongiovanni, J. L. Staehli, and B. Deveaud, High-temperature ultrafast polariton parametric amplification in semiconductor microcavities, Nature (London) 414, 731 (2001).

[8] R. M. Stevenson, V. N. Astratov, M. S. Skolnick, D. M. Whittaker, M. Emam-Ismail, A. I. Tartakovskii, P. G. Savvidis, 
J. J. Baumberg, and J. S. Roberts, Continuous Wave Observation of Massive Polariton Redistribution by Stimulated Scattering in Semiconductor Microcavities, Phys. Rev. Lett. 85, 3680 (2000).

[9] J. Kasprzak, M. Richard, S. Kundermann, A. Baas, P. Jeambrun, J. M. J. Keeling, F. M. Marchetti, M. H. Szymańska, R. André, J. L. Staehli, V. Savona, P. B. Littlewood, B. Deveaud, and Le Si Dang, Bose-Einstein condensation of exciton polaritons. Nature (London) 443, 409 (2006).

[10] A. Amo, J. Lefrère, S. Pigeon, C. Adrados, C. Ciuti, I. Carusotto, R. Houdré, E. Giacobino, and A. Bramati, Superfluidity of polaritons in semiconductor microcavities, Nat. Phys. 5, 805 (2009).

[11] P. M. Walker, L. Tinkler, D. V. Skryabin, A. Yulin, B. Royall, I. Farrer, D. A. Ritchie, M. S. Skolnick, and D. N. Krizhanovskii, Ultra-low-power hybrid light-matter solitons, Nat. Commun. 6, 8317 (2015).

[12] T. C. H. Liew, A. V. Kavokin, and I. A. Shelykh, Optical Circuits Based on Polariton Neurons in Semiconductor Microcavities, Phys. Rev. Lett. 101, 016402 (2008).

[13] A. Amo, T. C. H. Liew, C. Adrados, R. Houdré, E. Giacobino, A. V. Kavokin, and A. Bramati, Exciton-polariton spin switches, Nat. Photonics 4, 361 (2010).

[14] C. Sturm, D. Tanese, H. S. Nguyen, H. Flayac, E. Galopin, A. Lemaître, I. Sagnes, D. Solnyshkov, A. Amo, G. Malpuech, and J. Bloch, All-optical phase modulation in a cavitypolariton Mach-Zehnder interferometer, Nat. Commun. 5, 3278 (2014).

[15] D. Sanvitto and S. Kéna-Cohen, The road towards polaritonic devices, Nat. Mater. 15, 1061 (2016).

[16] A. V. Zasedatelev, A. V. Baranikov, D. Urbonas, F. Scafirimuto, U. Scherf, T. Stöferle, R. F. Mahrt, and P. G. Lagoudakis, A room-temperature organic polariton transistor, Nat. Photonics 13, 378 (2019).

[17] C. Leblanc, G. Malpuech, and D. D. Solnyshkov, Highfrequency exciton-polariton clock generator, Phys. Rev. B 101, 115418 (2020).

[18] T. Ellenbogen and K. B. Crozier, Exciton-polariton emission from organic semiconductor optical waveguides, Phys. Rev. B 84, 161304(R) (2011).

[19] H. Takeda and K. Sakoda, Exciton-polariton mediated light propagation in anisotropic waveguides, Phys. Rev. B 86, 205319 (2012).

[20] S. Pirotta, M. Patrini, M. Liscidini, M. Galli, G. Dacarro, G. Canazza, G. Guizzetti, D. Comoretto, and D. Bajoni, Strong coupling between excitons in organic semiconductors and Bloch surface waves, Appl. Phys. Lett. 104, 051111 (2014).

[21] D. D. Solnyshkov, H. Terças, and G. Malpuech, Optical amplifier based on guided polaritons in $\mathrm{GaN}$ and $\mathrm{ZnO}$, Appl. Phys. Lett. 105, 231102 (2014).

[22] I. Rosenberg, Y. Mazuz-Harpaz, R. Rapaport, K. West, and L. Pfeiffer, Electrically controlled mutual interactions of flying waveguide dipolaritons, Phys. Rev. B 93, 195151 (2016).

[23] J. Ciers, J. G. Roch, J.-F. Carlin, G. Jacopin, R. Butté, and N. Grandjean, Propagating Polaritons in III-Nitride Slab Waveguides, Phys. Rev. Appl. 7, 034019 (2017).

[24] O. Jamadi, F. Reveret, P. Disseix, F. Medard, J. Leymarie, A. Moreau, D. Solnyshkov, C. Deparis, M. Leroux, E. Cambril, S. Bouchoule, J. Zuniga-Perez, and G. Malpuech, Edge-emitting polariton laser and amplifier based on a $\mathrm{ZnO}$ waveguide, Light: Sci. Appl. 7, 82 (2018).

[25] I. Rosenberg, D. Liran, Y. Mazuz-Harpaz, K. West, L. Pfeiffer, and R. Rapaport, Strongly interacting dipolar-polaritons, Sci. Adv. 4, eaat8880 (2018).

[26] S. Christopoulos, G. Baldassarri Höger von Högersthal, A. Grundy, P. G. Lagoudakis, A. V. Kavokin, J. J. Baumberg, G. Christmann, R. Butté, E. Feltin, J.-F. Carlin, and N. Grandjean, Room-Temperature Polariton Lasing in Semiconductor Microcavities, Phys. Rev. Lett. 98, 126405 (2007).

[27] G. Christmann, R. Butté, E. Feltin, J.-F. Carlin, and N. Grandjean, Room temperature polariton lasing in a GaN/AlGaN multiple quantum well microcavity, Appl. Phys. Lett. 93, 051102 (2008).

[28] F. Tassone, C. Piermarocchi, V. Savona, A. Quattropani, and P. Schwendimann, Bottleneck effects in the relaxation and photoluminescence of microcavity polaritons, Phys. Rev. B 56, 7554 (1997).

[29] F. Tassone and Y. Yamamoto, Exciton-exciton scattering dynamics in a semiconductor microcavity and stimulated scattering into polaritons, Phys. Rev. B 59, 10830 (1999).

[30] G. Malpuech, A. Di Carlo, A. Kavokin, J. J. Baumberg, M. Zamfirescu, and P. Lugli, Room-temperature polariton lasers based on GaN microcavities, Appl. Phys. Lett. 81, 412 (2002).

[31] J. Kasprzak, D. D. Solnyshkov, R. André, Le Si Dang, and G. Malpuech, Formation of an Exciton Polariton Condensate: Thermodynamic versus Kinetic Regimes, Phys. Rev. Lett. 101, 146404 (2008).

[32] J. Levrat, R. Butté, E. Feltin, J.-F. Carlin, N. Grandjean, D. Solnyshkov, and G. Malpuech, Condensation phase diagram of cavity polaritons in GaN-based microcavities: Experiment and theory, Phys. Rev. B 81, 125305 (2010).

[33] O. Jamadi, F. Réveret, E. Mallet, P. Disseix, F. Médard, M. Mihailovic, D. Solnyshkov, G. Malpuech, J. Leymarie, X. Lafosse, S. Bouchoule, F. Li, M. Leroux, F. Semond, and J. Zuniga-Perez, Polariton condensation phase diagram in wideband-gap planar microcavities: GaN versus $\mathrm{ZnO}$, Phys. Rev. B 93, 115205 (2016).

[34] O. Jamadi, F. Réveret, D. Solnyshkov, P. Disseix, J. Leymarie, L. Mallet-Dida, C. Brimont, T. Guillet, X. Lafosse, S. Bouchoule, F. Semond, M. Leroux, J. Zuniga-Perez, and G. Malpuech, Competition between horizontal and vertical polariton lasing in planar microcavities, Phys. Rev. B 99, 085304 (2019).

[35] P. M. Walker, L. Tinkler, B. Royall, D. V. Skryabin, I. Farrer, D. A. Ritchie, M. S. Skolnick, and D. N. Krizhanovskii, Dark Solitons in High Velocity Waveguide Polariton Fluids, Phys. Rev. Lett. 119, 097403 (2017).

[36] P. M. Walker, C. E. Whittaker, D. V. Skryabin, E. Cancellieri, B. Royall, M. Sich, I. Farrer, D. A. Ritchie, M. S. Skolnick, and D. N. Krizhanovskii, Spatiotemporal continuum generation in polariton waveguides, Light: Sci. Appl. 8, 6 (2019).

[37] L. E. Tapia Rodriguez, P. M. Walker, H. Sigurdsson, B. Royall, I. Farrer, D. A. Ritchie, A. V. Yulin, I. A. Shelykh, M. S. Skolnick, and D. N. Krizhanovskii, Amplification of nonlinear polariton pulses in waveguides, Opt. Express 27, 10692 (2019).

[38] A. I. Tartakovskii, M. Emam-Ismail, R. M. Stevenson, M. S. Skolnick, V. N. Astratov, D. M. Whittaker, J. J. Baumberg, and J. S. Roberts, Relaxation bottleneck and its suppression 
in semiconductor microcavities, Phys. Rev. B 62, R2283(R) (2000).

[39] M. Glauser, C. Mounir, G. Rossbach, E. Feltin, J.-F. Carlin, R. Butté, and N. Grandjean, InGaN/GaN quantum wells for polariton laser diodes: Role of inhomogeneous broadening, J. Appl. Phys. 115, 233511 (2014).

[40] J. Ciers, G. Jacopin, G. Callsen, C. Bougerol, J.-F. Carlin, R. Butté, and N. Grandjean, Near-UV narrow bandwidth optical gain in lattice-matched III-nitride waveguides, Jpn. J. Appl. Phys. 57, 090305 (2018).

[41] G. Rossbach, J. Levrat, G. Jacopin, M. Shahmohammadi, J.-F. Carlin, J.-D. Ganière, R. Butté, B. Deveaud, and N. Grandjean, High-temperature Mott-transition in wide band-gap semiconductor quantum wells, Phys. Rev. B 90, 201308(R) (2014).

[42] F. Semond, I. R. Sellers, F. Natali, D. Byrne, M. Leroux, J. Massies, N. Ollier, J. Leymarie, P. Disseix, and A. Vasson, Strong light-matter coupling at room temperature in simple geometry GaN microcavities grown on silicon, Appl. Phys. Lett. 87, 021102 (2005).

[43] A. Kavokin and G. Malpuech, Cavity Polaritons (Elsevier, Amsterdam, The Netherlands, 2003).

[44] D. Solnyshkov, H. Ouerdane, and G. Malpuech, Kinetic phase diagrams of GaN-based polariton lasers, J. Appl. Phys. 103, 016101 (2008).

[45] R. Butté, G. Delalleau, A. I. Tartakovskii, M. S. Skolnick, V. N. Astratov, J. J. Baumberg, G. Malpuech, A. Di Carlo, A. V. Kavokin, and J. S. Roberts, Transition from strong to weak coupling and the onset of lasing in semiconductor microcavities, Phys. Rev. B 65, 205310 (2002).

[46] L. Pitaevskii and S. Stringari, Bose-Einstein Condensation (Oxford University Press, Oxford, 2003).

[47] D. D. Solnyshkov, H. Terças, K. Dini, and G. Malpuech, Hybrid Boltzmann-Gross-Pitaevskii theory of Bose-Einstein condensation and superfluidity in open driven-dissipative systems, Phys. Rev. A 89, 033626 (2014).

[48] D. M. Di Paola, P. M. Walker, R. P. A. Emmanuele, A. V. Yulin, J. Ciers, Z. Zaidi, J.-F. Carlin, N. Grandjean, I. Shelykh, M. S. Skolnick, R. Butté, and D. N. Krizhanovskii, Ultrafast-nonlinear ultraviolet pulse modulation in an AlIn$\mathrm{GaN}$ polariton waveguide operating up to room temperature, arXiv:2009.02059.

[49] G. Perillat-Merceroz, G. Cosendey, J.-F. Carlin, R. Butté, and N. Grandjean, Intrinsic degradation mechanism of nearly lattice-matched InAlN layers grown on GaN substrates, J. Appl. Phys. 113, 063506 (2013).

[50] Lumerical Solutions, Inc., FDTD Solutions v. 8.11.422.

[51] D. Brunner, H. Angerer, E. Bustarret, F. Freudenberg, R. Höpler, R. Dimitrov, O. Ambacher, and M. Stutzmann, Optical constants of epitaxial AlGaN films and their temperature dependence, J. Appl. Phys. 82, 5090 (1997).

[52] R. Butté, E. Feltin, J. Dorsaz, G. Christmann, J.-F. Carlin, N. Grandjean, and M. Ilegems, Recent progress in the growth of highly reflective nitride-based distributed Bragg reflectors and their use in microcavities, Jpn. J. Appl. Phys. 44, 7207 (2005). 